\title{
Long non-coding RNA HOTAIR promotes burn wound healing by regulating epidermal stem cells
}

\author{
YAN SHI $^{1,2}$, RONGHUA YANG ${ }^{3}$, LONGXIANG TU $^{1}$ and DEWU LIU ${ }^{1}$ \\ ${ }^{1}$ Burns Institute, The First Affiliated Hospital of Nanchang University, Nanchang, Jiangxi 330006; \\ ${ }^{2}$ Department of Plastic and Aesthetic Surgery, Jiangxi Maternal and Child Health Hospital, Nanchang, Jiangxi 330008; \\ ${ }^{3}$ Burns Department, The First People's Hospital of Foshan, Foshan, Guangdong 528000, P.R. China
}

Received December 14, 2019; Accepted May 27, 2020

DOI: $10.3892 / \mathrm{mmr} .2020 .11268$

\begin{abstract}
Local transplantation of epidermal stem cells (ESCs) exerts a therapeutic effect on burn wounds. However, cell viability can impede their clinical application. HOX antisense intergenic RNA (HOTAIR) is involved in regulating adult tissue stem cells, as well as in developmental patterning and pluripotency. However, little is known about its role in regulating ESCs. The present study was performed to investigate the effects of HOTAIR in the modulation of ESCs and wound repair. Firstly, reverse transcription-quantitative PCR was used to detect the relative expression of HOTAIR during burn wound healing in mice to determine whether HOTAIR is associated with wound healing. Subsequently, ESCs derived from mouse skin were transfected with a lentiviral vector to overexpress or knockdown HOTAIR. The effects of HOTAIR on cell proliferation and differentiation were measured by 5-bromodeoxyuridine and MTT assays, and by assessing NANOG mRNA expression. Lastly, mice with burns were administered a subcutaneous injection of HOTAIR-overexpressing ESCs. Images were captured and histological analyses were performed to evaluate wound healing. The results revealed that the expression of HOTAIR gradually increased and peaked at day 7 post-burn and maintained at relatively high levels until day 14 post-burn during
\end{abstract}

Correspondence to: Dr Dewu Liu, Burns Institute, The First Affiliated Hospital of Nanchang University, 17 Yongwaizheng Street, Nanchang, Jiangxi 330006, P.R. China

E-mail: dewuliu@126.com

Abbreviations: ASCs, adipose-derived stem cells; BrdU, 5-bromodeoxyuridine; CD, cluster of differentiation; ESCs, epidermal stem cells; H\&E, hematoxylin and eosin; HOTAIR, HOX antisense intergenic RNA; lncRNAs, long chain non-coding RNAs; MSCs, mesenchymal stem cells; NC, negative controls; RT-qPCR, reverse transcriptase quantitative polymerase chain reaction; UVB, ultraviolet B

Key words: epidermal stem cells, HOX antisense intergenic RNA, proliferation, differentiation, stemness, re-epithelialization, wound healing wound healing. Furthermore, overexpression of HOTAIR promoted ESC proliferation and maintained the stem cell state in vitro. By contrast, suppression of HOTAIR inhibited cell proliferation and cell stemness. It was also identified that HOTIR-overexpressing ESCs accelerated re-epithelialization and facilitated burn wound repair. In conclusion, the present findings confirmed an essential role of HOTAIR in the regulation of ESC proliferation and stemness. Therefore, targeting HOTAIR in ESCs may be a potentially promising therapy for burn wound healing.

\section{Introduction}

Burns are characterized by the presence of cutaneous tissue injury, excessive scarring and skin contracture. It is generally understood that the early closure of a burn wound is required to dramatically decrease mortality, the length of hospital stay and subsequent hypertrophic scarring via reducing the incidence of infection and fluid loss (1-4). Consequently, a vital issue of burn wound treatment is how to effectively promote wound closure.

The subcutaneous injection of allogeneic stem cells has been reported to promote wound healing and improve the quality of repair (5). The rapid advancement in stem cell studies has clearly demonstrated that stem cells possess a potential therapeutic ability to overcome limitations of the current treatments as it can accelerate wound healing with tissue regeneration. For example, administration of mesenchymal stem cells accelerates wound healing through the recruitment of macrophages and endothelial lineages in a paracrine manner (6). The effect of adipose-derived stem cells in wound healing is via the stimulation of neovascularization by paracrine factors (7). In addition, embryonic stem cells represent promising stem cell sources for wound repair through differentiating into keratinocytes in the presence of selected medium, including particular growth factors (8). Thus, the selection of a suitable stem cell is a challenge in order to achieve a desirable efficacy during wound healing.

Among numerous stem cells, epidermal stem cells (ESCs) are the preferred choice for therapeutic approaches in terms of burn wounds due to their high availability and lack of ethical concerns, such as those for embryonic stem cells. Relevant investigations also demonstrated that ESCs exert 
compensatory proliferation, followed by migration to the wound area and lastly directional differentiation to restore the wound in response to the injured skin (9-11). Thus, research investigating the methods that enhance the beneficial effect of ECSs on burn wound closure is required. Furthermore, cluster of differentiation (CD)44 has been identified as a surface marker of ESCs $(12,13)$ and has been reported to be important for epidermal homeostasis (14). The CD44+CD45-CD34-CD14population is one of the most used ESCs-enriched populations in the field of epidermal biology $(13,15,16)$. Therefore, flow cytometry was employed to detect the changes of cells markers (CD44, CD45, CD34 and CD14) to identify ESCs in the present study. Meanwhile, NANOG is a key regulatory factor for maintenance of the self-renewal and pluripotency of stem cells (17). As stem cells are induced to differentiate into other cells, the expression level of NANOG begins to decrease. Thus the mRNA expression of NANOG was detected to assess ESC stemness.

Long non-coding RNAs (lncRNAs) are defined as a class of non-protein coding RNAs (>200 nucleotides), and have been indicated to serve a critical role in modulating protein transcription (18) and regulating adult tissue stem cells, such as in the skin, muscle and brain, as well as in developmental patterning and pluripotency (19-21). Previous studies have indicated that numerous lncRNAs act as important regulators of the proliferation and directed differentiation of ESCs $(20,22,23)$. The lncRNA HOX antisense intergenic RNA (HOTAIR) is expressed by the HOXC locus on chromosome $12 \mathrm{q} 13.13$ and is involved in multiple human biological activities (24). Investigations have demonstrated that HOTAIR modulates the proliferation and directed differentiation of various cell types, such as dermal papilla cells and cutaneous squamous cells $(25,26)$. Indeed, increasing evidence has suggested that HOTAIR serves an important role in maintaining cell stemness (27-30). A recent study also revealed that increased HOTAIR levels were detected in ultraviolet B (UVB)-induced skin injury and were associated with sunburn wound healing; however, the specific mechanism governing this remain to be determined (31). Based on these findings, it was speculated that HOTAIR could be associated with the regulation of ESCs and consequently participate in burn wound healing.

\section{Materials and methods}

Animals. A total of 94 female BALB/c mice (age, 6-8 weeks; weight, 20-30 g) were purchased from Shanghai Laboratory Animal Center (Shanghai SLAC Laboratory Animal Co., Ltd.). The Animal Research Center of the First Affiliated Hospital of Nanchang University provided a temperature- and humidity-controlled condition $\left(24^{\circ} \mathrm{C}\right.$ with $12 \mathrm{~h}$ light/dark cycle, $35-40 \%$ humidity) and ad libitum access to a standard rodent diet and water (LabDiet-5001; Purina Mills, Inc.) for all mice. All animal experiments were conducted according to the standards of the 'Guide for the Care and Use of Laboratory Mice' (Institute of Laboratory Animal Resources, Commission on Life Sciences 2011) (32) and were approved by the Ethics Committee of the First Affiliated Hospital of Nanchang University. All experimental procedures were conducted and performed by experts who were blinded to the experiment conditions.
Mouse model of burn injury. The models of burn injury were established according to previous studies with minor modifications $(9,33)$. A total of 92 mice were anesthetized with $1 \%$ pentobarbital (30 mg/kg, intraperitoneally) and the hair on their back was shaved. Parameters of anesthesia including spontaneous breathing, blink reflex, muscle tension and reflex response were monitored. Then, a circular, burn cutaneous wound of $10 \mathrm{~mm}$ in diameter was made in the middle of the back using an $100^{\circ} \mathrm{C}$ electric copper plate tip. The copper plate tip was vertically pressed over the mouse skin for $10 \mathrm{sec}$ to produce burn injury and temperature of the copper plate tip was monitored and regulated by connection to an electronic temperature controller. Shortly afterwards, gauze pre-embedded in $22^{\circ} \mathrm{C}$ isotonic saline was applied to cover the wound for $5 \mathrm{~min}$ (34). Following conclusion of the procedure, the mice were returned to their individual cages for recovery at $24^{\circ} \mathrm{C}$ with $12 \mathrm{~h} \mathrm{light/dark} \mathrm{cycle} \mathrm{and} 35-40 \%$ humidity with free access to food and water. A total of $30 \mathrm{mg}$ codeine phosphate was added in $500 \mathrm{ml}$ water for analgesia for the $24 \mathrm{~h}$ after burn injury. The remaining 2 unburnt mice were used for the isolation and culture of mouse ESC.

$R N A$ extraction and reverse transcription-quantitative $P C R$ (RT-qPCR). Total RNA was isolated from the burnt skin tissue of 12 mice and the ESCs using TRIzol ${ }^{\circledR}$ reagent (Invitrogen; Thermo Fisher Scientific, Inc.). Total RNA (1 $\mu \mathrm{g})$ was converted into first strand complementary (c)DNA using a RT reagent kit (Invitrogen; Thermo Fisher Scientific, Inc.) at $42^{\circ} \mathrm{C}$ for $1 \mathrm{~h}$ according to the manufacturer's instructions. The conditions of qPCR using the SYBR Premix Ex Taq kit (Takara Bio, Inc.) were as follows: Initial denaturation for $5 \mathrm{~min}$ at $95^{\circ} \mathrm{C}$, then 40 cycles of denaturation at $94^{\circ} \mathrm{C}$ for $30 \mathrm{sec}$, annealing for $30 \mathrm{sec}$ at $56^{\circ} \mathrm{C}$, and elongation for $25 \mathrm{sec}$ at $72^{\circ} \mathrm{C}$. The primer sequences used were as follows: HOTAIR forward, 5'-GGTAGAAAAAGCAACCACGAA GG-3' and reverse, 5'-ACATAAACCTCTGTCTGTGAGTGC C-3'; NANOG forward, 5'-CCGTTGGGCTGACATGAG CGT-3' and reverse, 5'-GGCAGGCATCGGCGAGGAAT-3'; and GAPDH forward, 5'-AGAAGGCTGGGGCTCATTTG-3' and reverse, 5'-AGGGGCCATCCACAGTCTTC-3'. GAPDH was used to normalized HOTAIR and NANOG levels. The $2^{-\Delta \Delta \mathrm{Cq}}$ method was used to evaluate the relative expression of mRNA (35).

Isolation and culture of mouse ESCs. The present study established methods based on previous reports to isolate and culture ESCs $(11,36,37)$. Then 2 BALB/c female mice aged 8 weeks old that had not been burnt were selected. Mice were anesthetized with $1 \%$ pentobarbital $(30 \mathrm{mg} / \mathrm{kg}$, intraperitoneally) and the hair on their back was shaved. Then mice were sacrificed. Parameters of anesthesia such as spontaneous breathing, blink reflex, muscle tension and reflex response were monitored. Dorsal skin tissues of mice were carefully obtained and cut into small fragments $\left(\sim 0.3 \times 0.3 \mathrm{~cm}^{2}\right)$ and then placed in PBS overnight at $4^{\circ} \mathrm{C}$. Subsequently, the epidermal segments were detached from the dermal tissue and digested in $0.25 \%$ trypsin (cat. no. 25200-056; Gibco; Thermo Fisher Scientific, Inc.) for $20 \mathrm{~min}$ at $37^{\circ} \mathrm{C}$. Suspensions of the digested material were generated by adding DMEM (12100-046; Gibco; Thermo Fisher Scientific, Inc.) containing 10\% FBS 
(Gibco; Thermo Fisher Scientific, Inc.). Cells adhering to the flasks were then collected by removing the non-adherent cells and maintaining the adherent cells and cultured in K-SFM medium (cat. no. 17005-042; Gibco; Thermo Fisher Scientific, Inc.) with $5 \% \mathrm{CO}_{2}$ at $37^{\circ} \mathrm{C}$.

Flow cytometry. To demonstrate that the cells isolated and cultured were ESCs, flow cytometry was used to examine the ecto-50'-nucleotidase expression, including CD44, CD45, CD34 and CD14, on ESCs by a FC500 flow cytometer (Beckman Coulter, Inc.). For this analysis, $1 \times 10^{6}$ cells were suspended in $2 \%$ Bovine Serum Albumin and labeled with CD44-FITC, CD45-FITC, CD34-FITC and CD14-FITC (all from BD Biosciences) on ice for 30 min according to the manufacturer's instructions. Lastly, Coulter CXP version 2.1 (Beckman Coulter, Inc.) software was used to analyze the relevant results.

Transfection and generation of stable cell lines. PCR was used to amplify the mouse HOTAIR transcript variant 3 cDNA with the same PCR conditions as above. The viral production was conducted according to the Tronolab instructions (38). The 293FT cells (Invitrogen) were cultured in DMEM (Gibco) containing $10 \%$ fetal bovine serum (Gibco). TransIntro ${ }^{\circledR}$ EL Transfection Reagent (FT201, TransGen Biotech) were employed. The psPAX2 packing plasmid (concentration: $1 \mathrm{mg} / \mathrm{ml}$, Invitrogen) and the pMD2G envelope plasmid (concentration: $1 \mathrm{mg} / \mathrm{ml}$, Invitrogen) were then transfected into 293FT cells. After transfection for $72 \mathrm{~h}$, cell supernatant was collected and sub-packed at $4^{\circ} \mathrm{C}$ for the following experiments. The resultant lentivirus was used to infect the isolated ESC cells. pcDNA plasmids overexpressing HOTAIR were termed pc-HOTAIR. By contrast, control plasmids were termed pc-vector. Short hairpin $(\mathrm{sh}) \mathrm{RNA}(1,024 \mu \mathrm{g})$ that targeted HOTAIR mRNA expression were constructed into the pcDNA3.1 plasmid (Shanghai GenePharma Co., Ltd.) and were termed sh-HOTAIR. The above HOTAIR-shRNA sequences was follows: 5'-TCGCTGTACTGTACACACCGC AGCGGGTACAGCGTTTC-3' (Shanghai GenePharma Co., Ltd.). Plasmids $(988 \mu \mathrm{g})$ carrying non-targeting sequence against HOTAIR (Shanghai GenePharma Co., Ltd.) were used as a negative control (NC) of sh-HOTAIR and termed sh-NC. The above NC-shRNA sequences was follows: 5'-AAAUCC AGAACCCUCUGACAUUUGA-3' (Shanghai GenePharma Co., Ltd.).

Lipofectamine ${ }^{\circledR} 3000$ reagent (Invitrogen; Thermo Fisher Scientific, Inc.) was used for transfection into mouse ESCs according to the manufacturer's protocols. In total, $80 \%$ of stably transfected cells were selected for further experiments using medium containing blasticidin (Invitrogen; Thermo Fisher Scientific, Inc.).

5-Bromodeoxyuridine (BrdU) incorporation assay. To determine the viability of ESCs, ESCs in each group were seeded in 24-well plates at a density of $2 \times 10^{4}$ cells per well (Sarstedt K.K.). Subsequently, $10 \mu 1$ BrdU (Sigma-Aldrich; Merck KGaA) solution containing $10 \mathrm{mM}$ BrdU dissolved in distilled PBS was added to each well to label proliferating cells, according to the manufacturer's instructions. Afterwards, each well was incubated with an anti-BrdU antibody (1:100;
BioLegend, http://www.biolegend.com/) at $4^{\circ} \mathrm{C}$ overnight and detected with a fluorescence-conjugated secondary antibody. Images of the stained cells were then obtained under a confocal laser scanning microscope at 100x magnification (FV1000, Olympus Corporation) and the percentage of BrdU-positive cells was calculated. The aforementioned experiments were performed in triplicate and measurements from five duplicate wells were used to generate the average results.

MTT assay. The proliferative ability of ESCs was determined by MTT assay (Sigma-Aldrich; Merck KGaA). ESCs in each group were seeded in 96-well plates at a density of $1 \times 10^{4}$ cells per well. At different time points $(24,48,72$ and $96 \mathrm{~h}$ after inoculation), $20 \mu \mathrm{l}$ MTT was added to each well and incubated for $3 \mathrm{~h}$ at $37^{\circ} \mathrm{C}$. The culture medium was then removed and formazan crystals were solubilized in $200 \mu \mathrm{l}$ DMSO. The optical density was measured at a wavelength of $490 \mathrm{~nm}$ by the use of a microplate reader (Molecular Devices, LLC).

Treatment of burn wound. To evaluate the effect of ESCs overexpressing HOTAIR on wound healing in vivo, 80 adult female BALB/c mice (age, 6-8 weeks; weight, 20-30 g) were purchased from Shanghai Laboratory Animal Center (Shanghai SLAC Laboratory Animal Co., Ltd.). The Animal Research Center of the First Affiliated Hospital of Nanchang University provided a temperature- and humidity-controlled condition $\left(24^{\circ} \mathrm{C}\right.$ with $12 \mathrm{~h}$ light/dark cycle, $35-40 \%$ humidity) and ad libitum access to a standard rodent diet and water (LabDiet-5001; Purina Mills, Inc.) for all mice. The mice were randomly divided into four groups and received intradermal injections of the following: i) HOTAIR-overexpressing ESCs (the pc-HOTAIR group); ii) ESCs transfected with a non-overexpressing HOTAIR sequence (the pc-vector group); iii) ESCs without lentivirus transfection (the con-ESCs group); and iv) physiological saline solution without ESCs (the control group). Subsequently, the model of burn injury was established using the protocol outlined in the "mouse model of burn injury' section above. Four injections of each treatment from four directions were administered around the wound $\left(0.8 \times 10^{6}\right.$ in $20 \mu \mathrm{l}$ of physiological saline solution per injection) in each group based on previous reports (10). The mice then returned to their individual cages at $24^{\circ} \mathrm{C}$ with $12 \mathrm{~h} \mathrm{light/dark} \mathrm{cycle}$ and $35-40 \%$ humidity with free access to food and water for the following experiments. Wound healing was evaluated on days $0,3,7$ and 14 post-injury. Images were captured to record wound healing and ImageJ software (version 1.46; National Institutes of Health) was used to evaluate the photographs in accordance with digital planimetry. Each measurement was repeated three times, with the average value taken as the experiments result. The percentage of wound closure was calculated according to previous reports $(9,10,37)$. It should be noted that the sign of wound closure was defined when the wound bed was re-epithelialized and covered with regenerated tissue.

Histological analysis. Mice used in the wound closure assessment were sacrificed at days $0,3,7$ and 14 post-burn injury and skin tissue samples were harvested using a $25 \mathrm{~mm}$ biopsy punch. The above mice were euthanized according to previous references $(9,10)$ with $1 \%$ pentobarbital $(80 \mathrm{mg} / \mathrm{kg}$, 
intraperitoneally). Subsequently, the entire cutaneous tissue samples were fixed using formalin $\left(10 \%\right.$ for $24 \mathrm{~h}$ at $\left.24^{\circ} \mathrm{C}\right)$ and embedded in paraffin. To evaluate the quality of wound healing, the embedded samples were sectioned to a $4 \mu \mathrm{m}$ thicknesses and further subjected to hematoxylin and eosin (H\&E) staining for $15 \mathrm{~min}$ at $35^{\circ} \mathrm{C}$. Standard light microscopy (Olympus Corporation) at 200x magnification was employed to assess $\mathrm{H} \& \mathrm{E}$ staining. Each slide was given a histological score ranging from 1-10 according to the following parameters: Re-epithelialization, wound bed cellularity, angiogenesis, granulation tissue formation and collagen deposition following previously established methods $(9,10)$ by two experts who were blinded to the experiment conditions.

Statistical analysis. All experiments were performed at least in triplicate. All experimental results are presented as the mean \pm standard deviation. The statistical analyses were conducted using SPSS 20.0 statistical software (IBM Corp.). $\mathrm{P}<0.05$ was considered to indicate a statistically significant difference. Prism version 5 (GraphPad Software, Inc.) was used to generate graphs and perform statistical analysis. P-values were calculated using one-way ANOVA or multifactorial ANOVA for comparisons between multiple groups. Bonferroni's post-hoc test was used for pairwise comparisons where appropriate.

\section{Results}

HOTAIR is enhanced during burn wound healing. To evaluate the expression levels of HOTAIR during burn wound healing, RT-qPCR was employed following the generation of a burn wound. The results revealed relatively low expression of HOTAIR at 0 days post-injury. As the wound healed, the expression of HOTAIR also gradually increased, HOTAIR expression peaked at day 7 post-injury and remained at a significantly higher level 14 days post-injury compared with the baseline level at 0 days post-injury (Fig. 1). This result suggested that HOTAIR is associated with burn wound healing.

Expression of HOTAIR in transfected ESCs. In order to investigate the regulative effects of HOTAIR in ESCs in vitro, ESCs were isolated following established methods as described previously $(11,36,37)$ and cultured. Cells in culture were distributed in clusters. The cell clusters were large and round, and the cells were predominantly large with a round nucleus and relatively little cytoplasm (Fig. 2A). These results were consistent with the morphological characteristics of ESCs (10). The cell type of ESCs that were isolated was further confirmed by overexpression of CD44 and lack of expression of CD45, CD34 and CD14 (Fig. 2B). RT-qPCR was used to observe HOTAIR expression levels in lentivirus-transfected ESCs. As presented in Fig. 2C, the mRNA expression levels of HOTAIR in the pc-HOTAIR group were significantly higher compared with those observed in the pc-vector group and the con-ESCs group $(\mathrm{P}<0.05)$. On the other hand, the results of RT-qPCR demonstrated that the expression of HOTAIR was significantly decreased in the sh-HOTAIR group compared with the sh-NC group and the con-ESCs group $(\mathrm{P}<0.05)$. The expression of HOTAIR was not significantly different among the pc-vector group, the con-ESCs group and the sh-NC group $(\mathrm{P}>0.05)$.

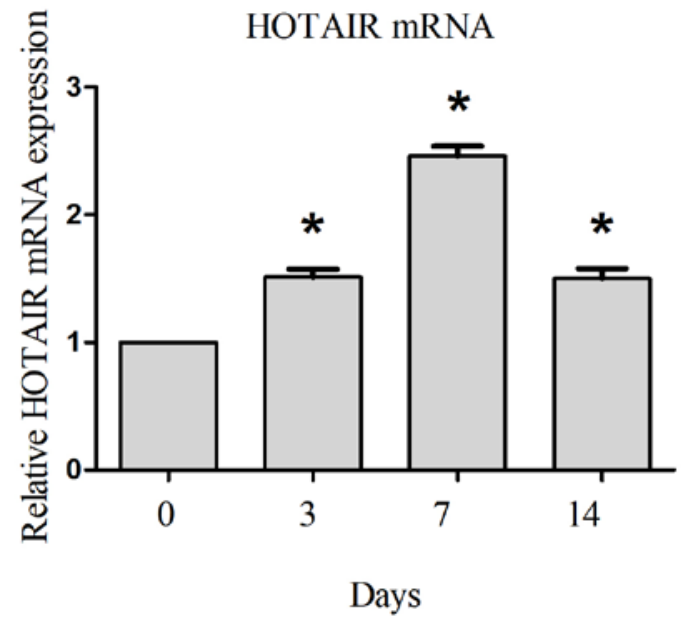

Figure 1. Expression of HOTAIR is elevated during wound healing. Values are reported as the mean \pm standard deviation from at least three independents. ${ }^{*} \mathrm{P}<0.05$ vs. baseline at day 0 post-injury. HOTAIR, HOX antisense intergenic RNA.

HOTAIR promotes the proliferative ability of ESCs and maintains stemness. Next, a BrdU incorporation assay and MTT assay were performed to evaluate the effect of HOTAIR on ESC proliferation (Fig. 3A and B). The results revealed that overexpression of HOTAIR in the pc-HOTAIR group significantly increased ESC proliferation compared with the pc-vector and con-ESCs groups, while suppression of HOTAIR in the sh-HOTAIR group significantly decreased the proliferative ability compared with the sh-NC and con-ESCs groups (both $\mathrm{P}<0.05$ ). In order to evaluate the effect of HOTAIR on ESC differentiation, NANOG mRNA expression levels were examined in five groups. The present study demonstrated that the mRNA expression of NANOG was significantly higher in the pc-HOTAIR group compared with the pc-vector and con-ESCs groups (Fig. $3 \mathrm{C} ; \mathrm{P}<0.05$ ), which suggests the overexpression of HOTAIR maintains the stem cell state. By contrast, suppression of HOTAIR in the sh-HOTAIR group significantly inhibited ESC stemness (Fig. 3C; $\mathrm{P}<0.05$ ).

ESCs with HOTAIR overexpression promotes burn wound healing. To investigate whether HOTAIR-overexpressing ESCs can affect burn wound healing in vivo, the present study transplanted ESCs overexpressing HOTAIR into mice wounds and observed wound healing. At day 7 post-burn, the newly generated epidermis partly covered the wounds and normal skin tissues of wound margins slightly contracted in the pc-HOTAIR group, which occurred earlier than that in the other three groups (Fig. 4A). The wounds of the pc-HOTAIR group healed quickly, and within this group, all wounds healed almost completely at day 14 post-burn, while a few wounds were unhealed in the con-ESCs and pc-vector groups, and the majority of wounds were unhealed in the control group at this timepoint (Fig. 4A). The further statistical analysis demonstrated that the ESC-treated burn wounds exhibited accelerated healing in mice compared with physiological saline solution-treated wounds (without ESCs; Fig. 4B; P<0.05). The results also indicated that the wound healing rates were further increased in mice treated with ESCs with HOTAIR 
A

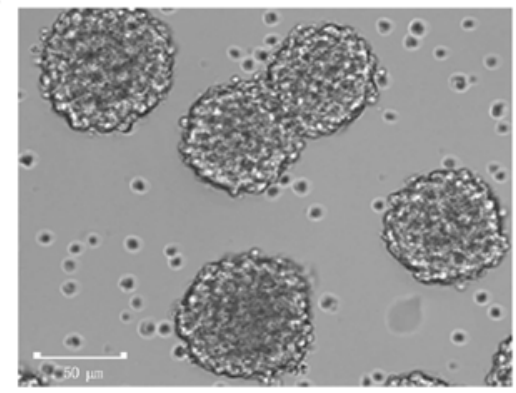

B
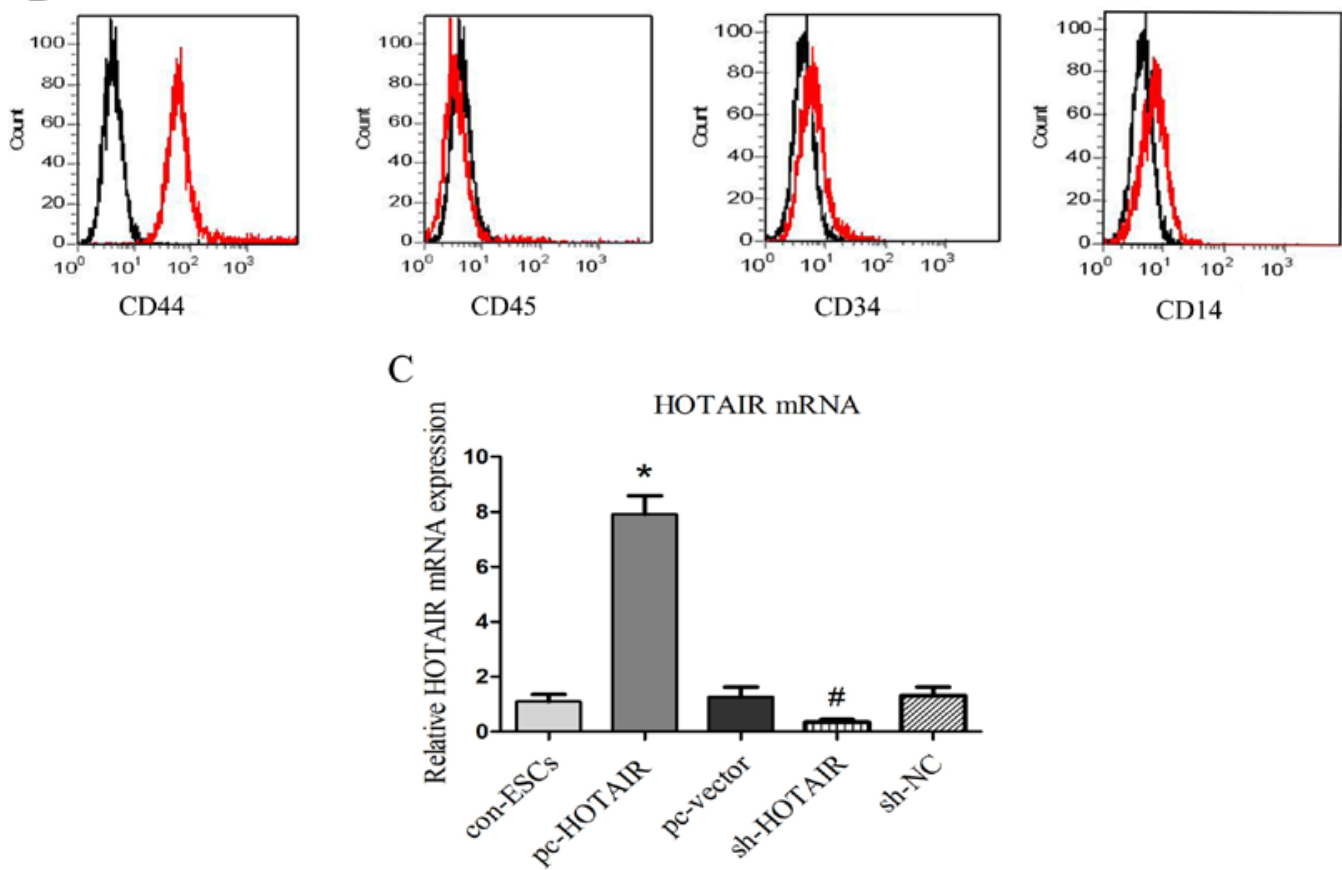

Figure 2. Expression of HOTAIR in transfected ESCs. (A) The typical morphology of ESCs. (B) The cell type of ESCs was confirmed by measuring the expression of the cell type surface markers CD44, CD45, CD34 and CD14, and quantifying the positively-stained cells. The flow cytometry histograms for selected cells markers from ESCs were displayed. The results of cells staining positive were indicated by display of the percentage. The red line indicates the positive staining cells, and the black line indicates the isotype-matched monoclonal antibody control. The results demonstrated positive surface staining for overexpression of CD44 and low expression of CD45, CD34 and CD14, which indicated that the isolated cells were ESCs. (C) Results revealed overexpression and suppression of HOTAIR in lentivirus-transfected ESCs. Values are presented as the mean \pm standard deviation from at least three independents. Scale bar $=50 \mu \mathrm{m}$. ${ }^{*} \mathrm{P}<0.05 \mathrm{pc}-\mathrm{HOTAIR}$ group vs. pc-vector and con-ESCs groups. ${ }^{\text {} P}<0.05$ sh-HOTAIR group vs. the sh-NC and con-ESCs groups. HOTAIR, HOX antisense intergenic RNA; ESC, epidermal stem cell; NC, negative control; sh, short hairpin RNA; CD, cluster of differentiation; pc, overexpression plasmid; con, parental control cells.

overexpression (the pc-HOTAIR group) compared with ESCs without lentivirus transfection (the con-ESCs group) and ESCs with blank lentivirus transfection (the pc-vector group; Fig. 4B; $\mathrm{P}<0.05)$.

The present study further performed histological evaluation in four treatment groups of mice at days $0,3,7$ and 14 post-burn $(n=5)$. Histological examination revealed the following: i) A large number of epidermal cells were detected in the pc-HOTAIR group at 7 days post-burn, while proliferation of few epidermal cells was detected in the con-ESCs and pc-vector groups, and necrotic tissue was observed in the control group. ii) The newly generated epidermal cells covered all wounds in the pc-HOTAIR group at 14 days post-burn, while the aforementioned cells covered most of the wounds in the con-ESCs and pc-vector groups, and the newly generated epidermal cells covered part of the wounds in the control group. These results indicated enhanced re-epithelialization in burn wounds treated with ESCs (the con-ESCs group) compared with physiological saline solution-treated wounds without ESCs (the control group; Fig. 5A). In addition, further results demonstrated that wounds in the pc-HOTAIR group exhibited markedly increased re-epithelialization compared with the three other groups (Fig. 5A). As expected, the histological scores at 7 and 14 days post-burn were significantly increased in the pc-HOTAIR group compared with the three other groups, which was consistent with the aforementioned results (Fig. 5B). These findings suggest the functional role of HOTAIR in mediating re-epithelialization to promote burn wound healing in vivo.

\section{Discussion}

lncRNAs are a fairly novel category of ncRNAs that exert important functions in maintaining cutaneous structure and 
A

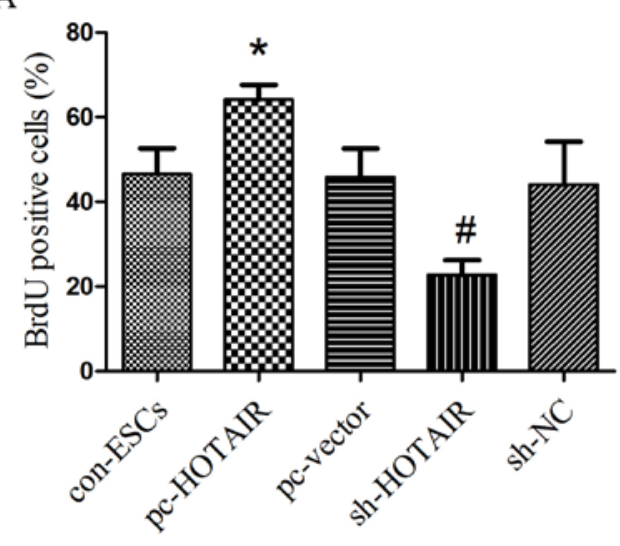

B

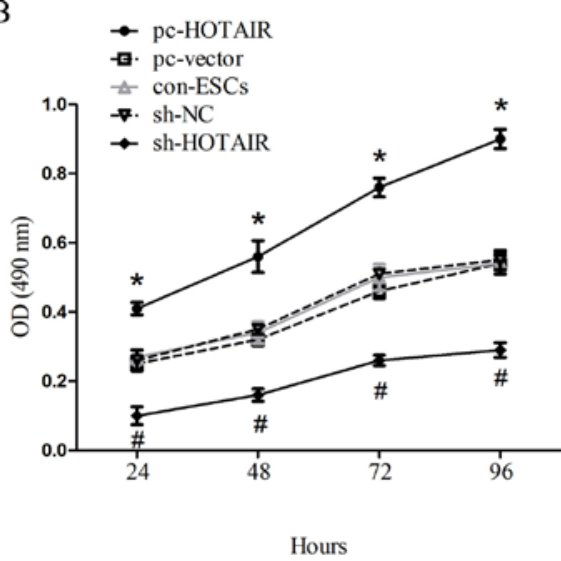

$\mathrm{C}$

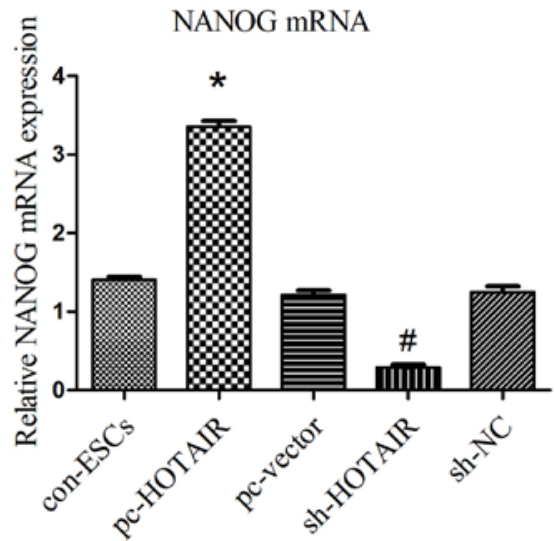

Figure 3. Functional role of HOTAIR during the proliferation and differentiation of ESCs. (A) Cell proliferation was examined by BrdU incorporation assay. (B) Cell proliferation was examined by MTT assay. (C) The mRNA expression of NANOG was upregulated in the pc-HOTAIR group and the differentiation potential was suppressed. Values are presented as the mean \pm standard deviation from at least three independents. "P $<0.05$ pc-HOTAIR group vs. pc-vector and con-ESCs groups. ${ }^{~} \mathrm{P}<0.05$ sh-HOTAIR group vs. sh-NC and con-ESCs groups. HOTAIR, HOX antisense intergenic RNA; ESC, epidermal stem cell; $\mathrm{NC}$, negative control; sh, short hairpin RNA; con, parental control cells; pc, overexpression plasmid; BrdU, 5-bromodeoxyuridine.

A Days
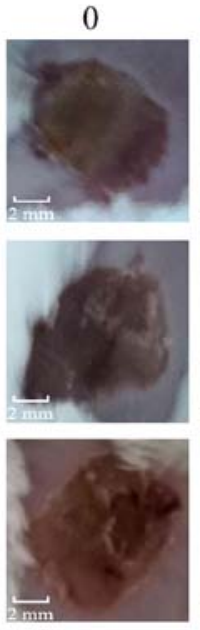

Control

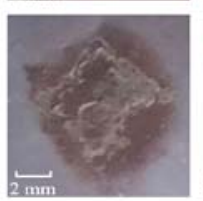

3
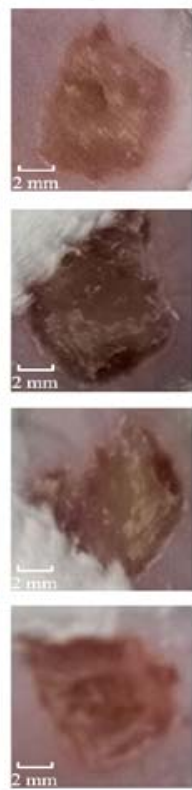

7
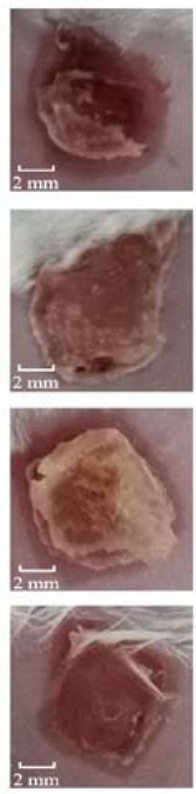

14
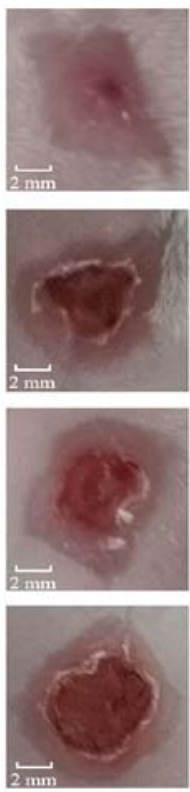

B

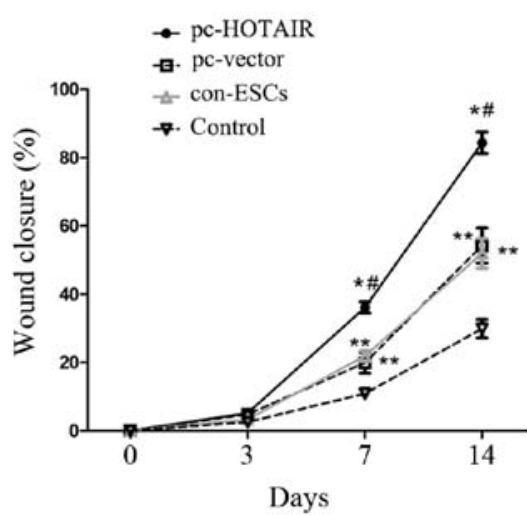

Figure 4. Effects of treatment with ESCs on burn wound (n=5). (A) The representative images of burn wounds in mice at 0,3,7 and 14 days post-burn. (B) The measurement of burn wound healing in four treatment groups as a percentage of wound closure. Values are presented as the mean \pm standard deviation. ${ }^{*} \mathrm{P}<0.05$ pc-HOTAIR group vs. pc-vector and con-ESCs groups. ${ }^{*} \mathrm{P}<0.05$ pc-HOTAIR group vs. control group (physiological saline solution without ESCs). ${ }^{* *} \mathrm{P}<0.05$ pc-vector group or the con-ESCs group vs. control group. HOTAIR, HOX antisense intergenic RNA; ESC, epidermal stem cell; pc, overexpression plasmid; con, parental control cells; d, days. 
A Days
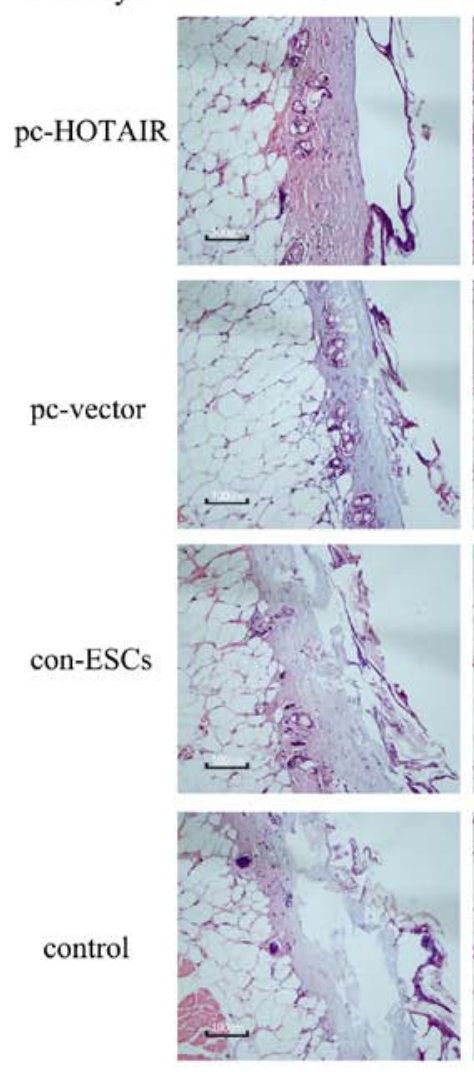

B
3
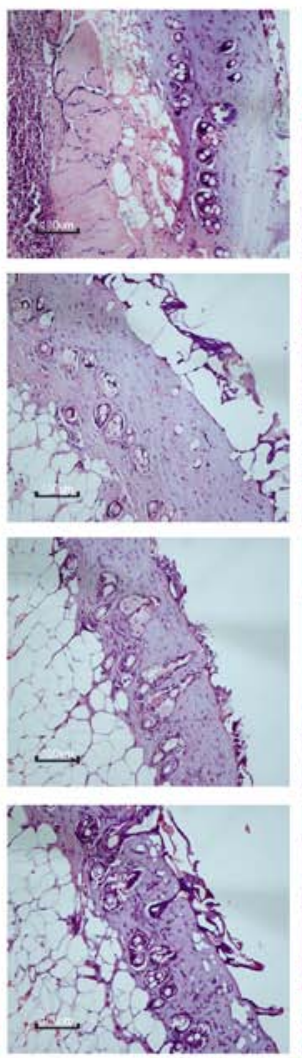

7
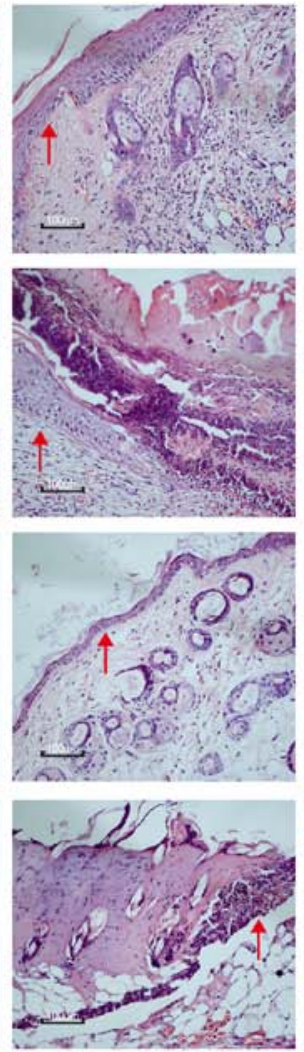

14
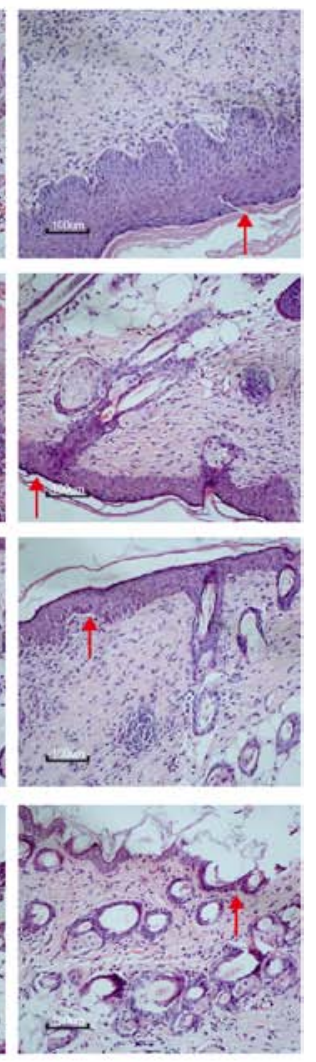

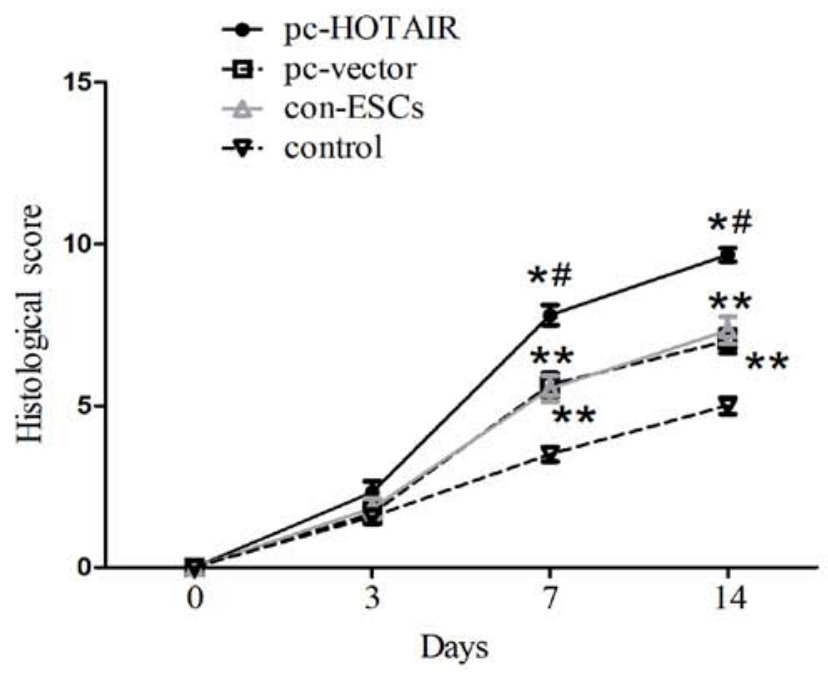

Figure 5. Histological analysis of burn wounds $(\mathrm{n}=5)$. (A) Representative images of wound histological staining at $0,3,7$ and 14 days post-burn. Scale bar $=100 \mu \mathrm{m}$. Arrowheads indicate the newly generated epidermis or wound surfaces. (B) Histological scores at 0, 3, 7 and 14 days post-burn. Values are presented as the mean \pm standard deviation. " $\mathrm{P}<0.05$ pc-HOTAIR group vs. pc-vector and con-ESCs groups. ${ }^{*} \mathrm{P}<0.05$ pc-HOTAIR group vs. control group (physiological saline solution without ESCs). ${ }^{* *} \mathrm{P}<0.05$ pc-vector group or the con-ESCs group vs. the control group. HOTAIR, HOX antisense intergenic RNA; ESC, epidermal stem cell; pc, overexpression plasmid; con, parental control cells; d, days.

function during processes such as wound repair $(19,39,40)$. Previously, HOTAIR IncRNA has received considerable attention in the regulation of cell growth, migration, differentiation and stemness in different tissues $(27,28)$. Numerous studies have indicated that HOTAIR is involved in biological activities of the skin (41-43). In dermal papilla cells, HOTAIR expression is associated with hairy gene regulation by activating the Wnt/ $\beta$-catenin signaling pathway $(42,43)$. Furthermore, the association between HOTAIR and UVB-induced cutaneous injury has been illustrated in previous studies (31). The current study also detected the expression changes of HOTAIR and the association with the process of burn wound healing in mice. Notably, the expression of HOTAIR gradually increased and peaked at 7 days post-injury during wound healing. Wound tissue repair mainly includes three stages: Inflammatory response stage, the proliferative stage and the remodeling 
stage. The proliferative stage occurs at 7 days post-injury, when re-epithelialization is the main marker accompanied by the formation of new blood vessels (44). The aforementioned results suggested that HOTAIR may serve a significant role in wound healing, particularly in re-epithelialization.

Re-epithelialization is a complicated and sophisticated process that involves essential biological processes of various cell types $(45,46)$. Numerous investigations have addressed the important effect of ESCs in promoting re-epithelialization (47-49). In response to cell loss in wounds, ESCs located in the proliferative basal cell layer participate in wound re-epithelialization by proliferation, differentiation and migration (50). On the other hand, increasing evidence has indicated that HOTAIR serves a key role in cell growth, differentiation and stemness (27-30). The present study further isolated ESCs according to established methods as reported previously $(11,36,37)$, and transfected them with lentivirus to deplete the expression of HOTAIR, thus determining whether HOTAIR is associated with the function of ESCs. The results demonstrated that HOTAIR contributes to the regulation of the proliferation and differentiation ability of ESCs. Overexpression of HOTAIR promoted ESC proliferation and maintained the stem cell state. By contrast, suppression of HOTAIR reduced cell proliferation and inhibited cell stemness. In light of previously published studies $(11,37)$, it was speculated that the Notch1 signaling pathway may contribute to the regulation of ESCs associated with HOTAIR. The Notch1 signaling pathway has previously been reported as a critical pathway in the determination of ESC proliferation, differentiation and stemness during wound healing. The activation of Notch1 signaling could upregulate ESC proliferation and maintain the multi-directional differentiation potential of ESCs with poor-differentiated status. Conversely, suppression of the Notch1 signaling pathway reduces ESC proliferation and induced ESCs to terminally differentiate, such as myofibroblasts. In addition, multiple studies have indicated that HOTAIR is involved in the regulation of Notch1 signaling in different malignant tissues (51-54). Overexpression of HOTAIR upregulates activation of the Notch1 signaling pathway to promote cervical cancer cell proliferation and invasion (53). Therefore, Notch1 signaling may contribute to ESC proliferation and stemness via activation of the Notch1 signaling pathway, which should be examined in future studies.

ESCs are the 'seed cells' in skin tissue engineering and are key functional cells during wound repair (55-57). ESCs are located in the basal layer of the epidermis, the outer root sheath of hair follicles and the base of sebaceous glands (50). ESCs only account for $1-10 \%$ of the total number of basal cells. Due to injury of the epidermis and dermis following a burn wound, the quantity and activity of ESCs in local wound tissues decreases accordingly. In recent years, local transplantation of ESCs has been shown to exert curative effects against burn wounds by making up for the reduced quantity and activity of ESCs on the local wound tissue $(58,59)$. In the present study, the isolation and ex vivo expansion of ESCs from skin was performed, and the subcutaneous injection of these ESCs resulted in accelerating wound healing, which is consistent with the aforementioned findings (60). Notably, CD44 was selected as a cell surface marker to identify these expanded ESCs due to the following reasons: i) CD44 has been well documented and applied for the identification of ESCs (12); ii) CD44 is a cell surface receptor of hyaluronate and CD44 knockout has been demonstrated to drive the epidermal atrophy in mouse epidermis $(14,61,62)$, which has been described as the phenotype of cutaneous insufficiency (63); iii) the CD44+ ${ }^{+}$ESCs exhibit enhanced self-renewal ability in vivo and in vitro, which is of high importance for medical applications of ESCs (13); and iv) although there is still no direct evidence about the association between HOTAIR with CD44 in skin tissue, it has been reported that HOTAIR impacts $\alpha 1,3$-fucosylated CD44 in other tissues, such as colorectal tissue (64).

Based on this information, mice with burn skin wounds were intradermally injected using ESCs with HOTAIR-overexpression to determine wound healing. Further results indicated that local transplantation of ESCs with HOTAIR-overexpression upregulates wound closure rates in mice, accompanied with acceleration of re-epithelialization in wounds in the pc-HOTAIR group compared with the con-ESCs group in vivo. The present study also suggests that enhancement of ESC proliferation and stemness via HOTAIR could facilitate re-epithelialization and wound repair. Similar to the results of the current study, previous studies have also indicated that suppression of ESC proliferation contributes to the inhibition of wound repair and cell compression of the leading edge toward the wound center $(65,66)$. The present study did not detect the effect of depletion of HOTAIR on ESCs in vivo experiment for the following reasons: i) During the design of the experiments it was concluded that knockdown of HOTAIR on ESCs would inhibit wound healing; and,ii) the main concern of the present study was to detect the effect of HOTIR-overexpressed ESCs for wound healing. Through observing and recording wound healing status and calculating the wound healing rates, the above results revealed the effects of overexpression of HOTAIR on ESCs wound closure rate. In further research, the effect of depletion of HOTAIR on ESCs in vivo should be detected. Besides that, the whole skin wounds at various stages should be harvested and analyzed by western blot and the related histological samples should be stained to detect the presence of proliferative markers. It is hypothesized that the aforementioned experimental results could be helpful in understanding the detailed processes of wound healing and exploring the mechanism of action of ESCs overexpressing HOTAIR during wound healing. A limitation of the present study was the traditional subcutaneous injection of allogeneic ESCs, which was associated with low cell viability and a high cell attrition rate in a previous experiment (67). Therefore, the treatment may be more suitable for superficial or small wounds rather than deep or large wounds (68), which partly interrupt the potential clinical application. For this, the experimental design requires further improvement.

In conclusion, the present study demonstrated that wound tissues have elevated HOTAIR levels during repair. Enhanced HOTAIR expression was indicated to be associated with ESC proliferation and stemness maintenance in vitro. Furthermore, the results indicated that HOTAIR could promote wound healing by facilitating stem cell therapeutics in vivo. This suggests that HOTAIR may be a potential therapeutic target in the promotion of burn wound healing via modulating ESCs. 


\section{Acknowledgements}

Not applicable.

\section{Funding}

The present study was supported by the National Natural Science Foundation of China (grant no. 81460293).

\section{Availability of data and materials}

The datasets used and/or analyzed during the present study are available from the corresponding author on reasonable request.

\section{Authors' contributions}

YS designed the study, performed experiments, analyzed the data and wrote the manuscript. RY performed experiments, analyzed the data. LT collected data and searched the literature. DL contributed significantly to the concept and design of the present study and wrote the manuscript. All authors have read and approved the final manuscript.

\section{Ethics approval and consent to participate}

All animal experiments were performed according to the Guide for the Care and Use of Laboratory Animals and were approved by the Ethics Committee of the First Affiliated Hospital of Nanchang University (approval no. 81460293). All efforts were made to minimize animal suffering and the number of animals used.

\section{Patient consent for publication}

Not applicable.

\section{Competing interests}

The authors declare that they have no competing interests.

\section{References}

1. Xiao-Wu W, Herndon DN, Spies M, Sanford AP and Wolf SE: Effects of delayed wound excision and grafting in severely burned children. Arch Surg 137: 1049-1054, 2002.

2. Kirn DS and Luce EA: Early excision and grafting versus conservative management of burns in the elderly. Plast Reconstr Surg 102: 1013-1017, 1998.

3. Saaiq M,Zaib S and Ahmad S: Early excision and grafting versus delayed excision and grafting of deep thermal burns up to $40 \%$ total body surface area: A comparison of outcome. Ann Burns Fire Disasters 25: 143-147, 2012.

4. Ong YS, Samuel $M$ and Song C: Meta-analysis of early excision of burns. Burns 32: 145-150, 2006.

5. Wang P, Hu Z, Cao X, Huang S, Dong Y, Cheng P, Xu H, Shu B, Xie J, Wu J, et al: Fibronectin precoating wound bed enhances the therapeutic effects of autologous epidermal basal cell suspension for full-thickness wounds by improving epidermal stem cells' utilization. Stem Cell Res Ther 10: 154, 2019.

6. Chen L, Tredget EE, Wu PY and Wu Y: Paracrine factors of mesenchymal stem cells recruit macrophages and endothelial lineage cells and enhance wound healing. PLoS One 3: e1886, 2008.

7. Elman JS, Li M, Wang F, Gimble JM and Parekkadan B: A comparison of adipose and bone marrow-derived mesenchymal stromal cell secreted factors in the treatment of systemic inflammation. J Inflamm (Lond) 11: 1, 2014
8. Aberdam D: Derivation of keratinocyte progenitor cells and skin formation from embryonic stem cells. Int J Dev Biol 48: 203-206, 2004.

9. Yang R, Wang J, Zhou Z, Qi S, Ruan S, Lin Z, Xin Q, Lin Y, Chen $X$ and Xie J: Curcumin promotes burn wound healing in mice by upregulating caveolin-1 in epidermal stem cells. Phytother Res 33: 422-430, 2019.

10. Yang R, Wang J, Zhou Z, Qi S, Ruan S, Lin Z, Xin Q, Lin Y, Chen X and Xie J: Role of caveolin-1 in epidermal stem cells during burn wound healing in rats. Dev Biol 445: 271-279, 2019.

11. Wang P, Shu B, Xu Y, Zhu J, Liu J, Zhou Z, Chen L, Zhao J, Liu X, Qi S, et al: Basic fibroblast growth factor reduces scar by inhibiting the differentiation of epidermal stem cells to myofibroblasts via the Notch1/Jagged1 pathway. Stem Cell Res Ther 8: 114, 2017.

12. Liu AY, True LD, LaTray L, Nelson PS, Ellis WJ, Vessella RL, Lange PH, Hood L and van den Engh G: Cell-cell interaction in prostate gene regulation and cytodifferentiation. Proc Natl Acad Sci USA 94: 10705-10710, 1997.

13. Szabo AZ, Fong S, Yue L, Zhang K, Strachan LR, Scalapino K, Mancianti ML and Ghadially R: The CD44+ ALDH+ population of human keratinocytes is enriched for epidermal stem cells with long-term repopulating ability. Stem Cells 31: 786-799, 2013.

14. Barnes L, Ino F, Jaunin F, Saurat JH and Kaya G: Inhibition of putative hyalurosome platform in keratinocytes as a mechanism for corticosteroid-induced epidermal atrophy. J Invest Dermatol 133: 1017-1026, 2013.

15. Barnes L, Saurat JH and Kaya G: Senescent atrophic epidermis retains Lrig1+ stem cells and loses Wnt signaling, a phenotype shared with CD44KO mice. PLoS One 12: e0169452, 2017.

16. Yang RH, Qi SH, Shu B, Ruan SB, Lin ZP, Lin Y, Shen R, Zhang FG, Chen XD and Xie JL: Epidermal stem cells (ESCs) accelerate diabetic wound healing via the Notch signalling pathway. Biosci Rep 36: e00364, 2016.

17. Zhao X, Wang F and Hou M: Expression of stem cell markers nanog and PSCA in gastric cancer and its significance. Oncol Lett 11: 442-448, 2016.

18. Da Sacco L, Baldassarre A and Masotti A: Bioinformatics tools and novel challenges in long non-coding RNAs (lncRNAs) functional analysis. Int J Mol Sci 13: 97-114, 2012.

19. Flynn RA and Chang HY: Long noncoding RNAs in cell-fate programming and reprogramming. Cell Stem Cell 14: 752-761, 2014.

20. Si Y, Bai J, Wu J, Li Q, Mo Y, Fang R and Lai W: IncRNA PlncRNA-1 regulates proliferation and differentiation of hair follicle stem cells through TGF- $\beta 1$-mediated Wnt $/ \beta$-catenin signal pathway. Mol Med Rep 17: 1191-1197, 2018.

21. Tian X, Tian J, Tang X, Ma J and Wang S: Long non-coding RNAs in the regulation of myeloid cells. J Hematol Oncol 9: 99, 2016.

22. Li J and Sen GL: Post-transcriptional mechanisms regulating epidermal stem and progenitor cell self-renewal and differentiation. J Invest Dermatol 136: 746-752, 2016.

23. Tanis SEJ, Köksal ES, van Buggenum JAGL and Mulder KW: BLNCR is a long non-coding RNA adjacent to integrin beta-1 that is rapidly lost during epidermal progenitor cell differentiation. Sci Rep 9: 31, 2019.

24. Somarowthu S, Legiewicz M, Chillón I, Marcia M, Liu F and Pyle AM: HOTAIR forms an intricate and modular secondary structure. Mol Cell 58: 353-561, 2015.

25. Sannigrahi MK, Sharma R, Panda NK and Khullar M: Role of non-coding RNAs in head and neck squamous cell carcinoma: A narrative review. Oral Dis 24: 1417-1427, 2018.

26. Sand M, Bechara FG, Sand D, Gambichler T, Hahn SA, Bromba M, Stockfleth E and Hessam S: Expression profiles of long noncoding RNAs in cutaneous squamous cell carcinoma. Epigenomics 8: 501-518, 2016.

27. Pádua Alves C,Fonseca AS, Muys BR, de Barros E Lima Bueno R, Burger MC, de Souza JE, Valente V, Zago MA and Silva WA Jr: Brief report: The lncRNA Hotair is required for epithelial-to-mesenchymal transition and stemness maintenance of cancer cell lines. Stem Cells 31: 2827-2832, 2013.

28. Sorensen KP, Thomassen M, Tan Q, Bak M, Cold S, Burton M, Larsen MJ and Kruse TA: Long non-coding RNA HOTAIR is an independent prognostic marker of metastasis in estrogen receptor-positive primary breast cancer. Breast Cancer Res Treat 142: 529-536,2013.

29. Liu T, Zhang H, Zheng J, Lin J, Huang Y, Chen J, Yu Z, Guo L, Pan W, Xiong Y and Chen C: SPION-mediated miR-141 promotes the differentiation of HuAESCs into dopaminergic neuron-like cells via suppressing lncRNA-HOTAIR. J Cell Mol Med 22: 2299-2310, 2018 
30. Gao S, Zhou B, Li H, Huang X, Wu Y, Xing C, Yu X and Ji Y: Long noncoding RNA HOTAIR promotes the self-renewal of leukemia stem cells through epigenetic silencing of p15. Exp Hematol 67: 32-40 e3, 2018.

31. Liu G and Zhang W: Long non-coding RNA HOTAIR promotes UVB-induced apoptosis and inflammatory injury by up-regulation of PKR in keratinocytes. Braz J Med Biol Res 51: e6896, 2018.

32. National Research Council (US) Committee for the Update of the Guide for the Care and Use of Laboratory Animals. 8th edition Washington, (DC): National Academies Press (US); 2011.

33. Zhu H, Wei X, Bian K and Murad F: Effects of nitric oxide on skin burn wound healing. J Burn Care Res 29: 804-814, 2008.

34. Calum H, Høiby N and Moser C: Burn mouse models. Methods Mol Biol 1149: 793-802, 2014.

35. Livak KJ and Schmittgen TD: Analysis of relative gene expression data using real-time quantitative PCR and the 2(-Delta Delta C(T)) method. Methods 25: 402-408, 2001

36. Liu Z, Xu Y, Chen L, Xie J, Tang J, Zhao J, Shu B, Qi S, Chen J, Liang G, et al: Dendritic epidermal T cells facilitate wound healing in diabetic mice. Am J Transl Res 8: 2375-2384, 2016

37. Shi Y, Shu B, Yang R, Xu Y, Xing B, Liu J, Chen L, Qi S, Liu X, Wang $\mathrm{P}$, et al: Wnt and Notch signaling pathway incolved in wound healing by targeting c-Myc and Hes1 separately. Stem Cell Res Ther 6: 120, 2015.

38. Bendiks L, Geiger F, Gudermann T, Feske S and Dietrich A: Store-operated $\mathrm{Ca}^{2+}$ entry in primary murine lung fibroblasts is independent of classical transient receptor potential (TRPC) channels and contributes to cell migration. Sci Rep 10: 6812, 2020.

39. Li D, Kular L, Vij M, Herter EK, Li X, Wang A, Chu T, Toma MA, Zhang L, Liapi E, et al: Human skin long noncoding RNA WAKMAR1 regulates wound healing by enhancing keratinocyte migration. Proc Natl Acad Sci USA 116: 9443-9452, 2019

40. Herter EK and Xu Landén N: Non-Coding RNAs: New players in skin wound healing. Adv Wound Care (New Rochelle) 6: 93-107, 2017.

41. Wan DC and Wang KC: Long noncoding RNA: Significance and potential in skin biology. Cold Spring Harb Perspect Med 4: a015404, 2014

42. Ge XS, Ma HJ, Zheng XH, Ruan HL, Liao XY, Xue WQ, Chen YB, Zhang Y and Jia WH: HOTAIR, a prognostic factor in esophageal squamous cell carcinoma, inhibits WIF-1 expression and activates Wnt pathway. Cancer Sci 104: 1675-1682, 2013.

43. Lin CM, Liu Y, Huang K, Chen XC, Cai BZ, Li HH, Yuan YP Zhang $\mathrm{H}$ and $\mathrm{Li} \mathrm{Y}$ : Long noncoding RNA expression in dermal papilla cells contributes to hairy gene regulation. Biochem Biophys Res Commun 453: 508-514, 2014.

44. Rousselle P, Montmasson M and Garnier C: Extracellular matrix contribution to skin wound re-epithelialization. Matrix Biol 75-76: 12-26, 2019

45. Gurtner GC, Werner S, Barrandon Y and Longaker MT: Wound repair and regeneration. Nature 453: 314-321, 2008.

46. Jacinto A, Martinez-Arias A and Martin P: Mechanisms of epithelial fusion and repair. Nat Cell Biol 3: E117-E123, 2001.

47. Gallico GG III, O'Connor NE, Compton CC, Kehinde O and Green $\mathrm{H}$ : Permanent coverage of large burn wounds with autologous cultured human epithelium. N Engl J Med 311: 448-451, 1984

48. Carsin H, Ainaud P, Le Bever H, Rives J, Lakhel A, Stephanazzi J, Lambert F and Perrot J: Cultured epithelial autografts in extensive burn coverage of severely traumatized patients: A five year single-center experience with 30 patients. Burns 26: 379-387, 2000.

49. De Luca M, Pellegrini G and Green H: Regeneration of squamous epithelia from stem cells of cultured grafts. Regen Med 1: 45-57, 2006.

50. Tumbar T, Guasch G, Greco V, Blanpain C, Lowry WE, Rendl M and Fuchs E: Defining the epithelial stem cell niche in skin. Science 303: 359-363, 2004.
51. Shao T, Hu Y, Tang W, Shen $\mathrm{H}, \mathrm{Yu} \mathrm{Z}$ and $\mathrm{Gu} \mathrm{J}$ : The long noncoding RNA HOTAIR serves as a microRNA-34a-5p sponge to reduce nucleus pulposus cell apoptosis via a NOTCH1-mediated mechanism. Gene 715: 144029, 2019.

52. Dong C, Liu S, Lv Y, Zhang C, Gao H, Tan L and Wang H: Long non-coding RNA HOTAIR regulates proliferation and invasion via activating Notch signalling pathway in retinoblastoma. J Biosci 41: 677-687, 2016.

53. Lee M, Kim HJ, Kim SW, Park SA, Chun KH, Cho NH, Song YS and Kim YT: The long non-coding RNA HOTAIR increases tumour growth and invasion in cervical cancer by targeting the Notch pathway. Oncotarget 7: 44558-44571, 2016.

54. Luo H, Liu WH, Liang HY, Yan HT, Lin N, Li DY, Wang T and Tang LJ: Differentiation-inducing therapeutic effect of Notch inhibition in reversing malignant transformation of liver normal stem cells via MET. Oncotarget 9: 18885-18895, 2018.

55. Morasso MI and Tomic-Canic M: Epidermal stem cells: The cradle of epidermal determination, differentiation and wound healing. Biol Cell 97: 173-183, 2005

56. Ito $\mathrm{M}$ and Cotsarelis $\mathrm{G}$ : Is the hair follicle necessary for normal wound healing? J Invest Dermatol 128: 1059-1061, 2008.

57. Garcin CL and Ansell DM: The battle of the bulge: Re-evaluating hair follicle stem cells in wound repair. Exp Dermatol 26: 101-104, 2017.

58. Chen M, Przyborowski M and Berthiaume F: Stem cells for skin tissue engineering and wound healing. Crit Rev Biomed Eng 37: 399-421, 2009.

59. Kanji S and Das H: Advances of stem cell therapeutics in cutaneous wound healing and regeneration. Mediators Inflamm 2017: 5217967, 2017.

60. Pashoutan Sarvar D, Shamsasenjan K and Akbarzadehlaleh P: Mesenchymal stem cell-derived exosomes: New opportunity in cell-free therapy. Adv Pharm Bull 6: 293-299, 2016.

61. Kaya G, Tran C, Sorg O, Hotz R, Grand D, Carraux P, Didierjean L, Stamenkovic I and Saurat JH: Hyaluronate fragments reverse skin atrophy by a CD44-dependent mechanism. PLoS Med 3: e493, 2006.

62. Kaya G and Saurat JH: Dermatoporosis: A chronic cutaneous insufficiency/fragility syndrome. Clinicopathological features, mechanisms, prevention and potential treatments. Dermatology 215: 284-294, 2007.

63. Barnes L, Puenchera J, Saurat JH and Kaya G: Lrig1 and CD44v3 expression in the human folliculosebaceous unit. Dermatology 231: 116-118, 2015.

64. Pan S, Liu Y, Liu Q, Xiao Y, Liu B, Ren X, Qi X, Zhou H, Zeng C and Jia L: HOTAIR/miR-326/FUT6 axis facilitates colorectal cancer progression through regulating fucosylation of CD44 via PI3K/AKT/mTOR pathway. Biochim Biophys Acta Mol Cell Res 1866: 750-760, 2019.

65. Aragona M, Dekoninck S, Rulands S, Lenglez S, Mascre G, Simons BD and Blanpain C: Defining stem cell dynamics and migration during wound healing in mouse skin epidermis. Nat Commun 8: 14684, 2017.

66. Dekoninck S and Blanpain C: Stem cell dynamics, migration and plasticity during wound healing. Nat Cell Biol 21: 18-24, 2019.

67. Kucharzewski M, Rojczyk E, Wilemska-Kucharzewska K, Wilk R, Hudecki J and Los MJ: Novel trends in application of stem cells in skin wound healing. Eur J Pharmacol 843: 307-315, 2019.

68. Yang R, Liu F, Wang J, Chen X, Xie J and Xiong K: Epidermal stem cells in wound healing and their clinical applications. Stem Cell Res Ther 10: 229, 2019.

This work is licensed under a Creative Commons Attribution-NonCommercial-NoDerivatives 4.0 International (CC BY-NC-ND 4.0) License. 